\title{
Molecular Expression Analysis of Restrictive Receptor for Interleukin 13, a Brain Tumor-associated Cancer/Testis Antigen
}

\author{
Waldemar Debinski and Denise M. Gibo \\ Section of Neurosurgery, Department of Surgery, Pennsylvania State University \\ College of Medicine, Hershey, Pennsylvania, U.S.A.
}

Accepted January 25, 2000.

\begin{abstract}
Background: The vast majority of patients with high-grade gliomas (HGG) over-express interleukin 4 (IL4)-independent binding sites for IL13 in situ. In addition, mutated IL13-based cytotoxins directed specifically toward glioma-associated sites are arguably the most active anti-glioma agents. Two IL13 receptor (R) proteins were identified: (1) IL13R $\alpha^{\prime}$, a component of the signaling, heterodimeric high-affinity receptor for IL13 that is shared with IL4, and (2) IL13R $\alpha$, a monomeric, IL4independent receptor.

Materials and Methods: We analyzed gene expression of IL13R $\alpha$, IL13R $\alpha^{\prime}$ and that of IL4R $R_{\beta}$, which is the other subunit of the shared IL13/4 receptor. The study was conducted with 40 human normal adult tissues, 20 discrete regions of the central nervous system (CNS), 7 fetal tissues, several cultured cell lines, and surgical CNS specimens.

Results: The most striking feature of the IL13R $\alpha$
\end{abstract}

gene expression was the virtual lack of its transcripts within the CNS. Furthermore, only the testes exhibited a prominent presence of the mRNA for IL13R $\alpha$ among peripheral organs. In contrast, the components of the shared IL13/4 receptor were readily detected both in the CNS and in vital organs, such as liver, heart, lungs, and gastrointestinal tract.

Conclusions. The results strongly support a need to redirect IL13 towards its more restrictive, IL4independent, receptor for glioma diagnosis and therapies. Moreover, the gene for IL $13 R \alpha$ resides on chromosome X. Since IL13R $\alpha$ is (1) a cancerassociated protein, (2) virtually restricted to testes among normal tissues, and (3) its gene is on chromosome $\mathrm{X}, \operatorname{IL13R} \alpha$ is unexpectedly categorized as a cancer/testis antigen. Our findings make IL13R $\alpha$ even more attractive as a target for variety of approaches in glioma molecular management.

\section{Introduction}

We documented the presence of a more restrictive receptor ( $R$ ) for interleukin 13 (IL13) in a vast majority of patients with high-grade glioma (HGG) in situ (1). Most of the cells within studied tumors appeared to express this receptor for IL13 (1). IL13 is an immune regulatory cytokine and acts similarly to its homologue, IL4 $(2,3)$. The major difference between

Address correspondence and reprints to: Waldemar Debinski, M.D., Ph.D., Section of Neurosurgery/H110, Department of Surgery, Pennsylvania State University College of Medicine, 500 University Drive, Hershey, PA 17033-0850, U.S.A.; Phone: 717-531-4541; Fax: 717531-5906; E-mail: wdebinski@psghs.edu the two is the lack of IL13-effect on T cells. IL13 and IL4 act in a similar manner because they share a receptor, IL13/4R, which transduces cellular signals and which was demonstrated in some normal tissues and adenocarcinomas $(4,5)$. This receptor is heterodimeric and is composed of $45-\mathrm{kDa}, \mathrm{ILI} 3 \mathrm{R} \alpha^{\prime}(6)$ and 140-kDa, IL4R $\mathrm{R}_{\beta}$ proteins (7; Fig. 1). The receptor for IL13 that is prevalent in HGG, however, is primarily IL4-independent and, consequently, termed more restrictive (1,8-10; Fig. 1). A $42-\mathrm{kDa}$ protein has been cloned, IL13R $\alpha$, which binds IL13 avidly by itself, but it has no affinity towards IL4 (11; Fig. 1).

Our laboratory conceived and designed IL13-based cytotoxins composed of IL13 and 
Shared IL13/4 Receptor

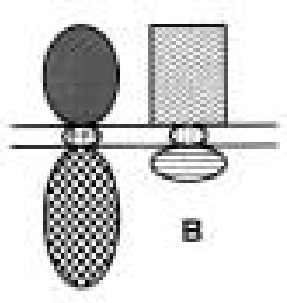

A
IL4-independent Receptor for IL13
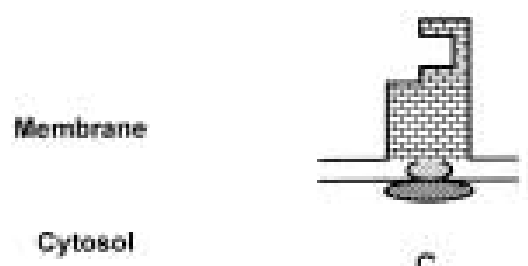

C

\section{A - $140-\mathrm{kDa}$ IL4 receptor $\beta$ chain \\ B - 45-kDa IL13Ra' (427 amino acids) also termed IL13Ra1 \\ C - 42-kDa IL13Ra (380 amino acids) also termed IL13R $\propto 2$}

Fig. 1. Model of two types of interleukin 13

(IL13) receptor. One is shared with IL4 physiological, heterodimeric IL13/4R and the other is an IL4independent monomeric, HGG-associated IL13R.

various genetically engineered derivatives of bacterial toxins, such as Pseudomonas exotoxin (PE) and Diphtheria toxin (DT; 1,4,9). Subsequently, we generated mutants of IL13 which do not signal through the shared IL13/4R, but which still do bind avidly the HGG-associated receptor $(12,13)$. Thus, we diverted the molecule of IL13 from its only known form of the physiological receptor and we were able to redirect IL13 to an IL4-independent receptor in gliomas. The mutants of IL13 fused to derivatives of bacterial toxins are extremely potent anti-glioma agents in vitro and in vivo (12). These drug candidates are in a preclinical stage of evaluation and will be tested in clinics in the near future. It is noteworthy that a prototype cytotoxic anti-HGG agent, a chemical conjugate of transferrin with derivative of DT, has proven to be clinically effective (14).

There are currently no studies that compare the expression of the two types of IL13 receptor in gliomas with an array of normal human tissues. Since IL13 cytotoxins are traveling the path from bench to clinic, we felt compelled to perform such a detailed analysis. It was important to verify whether or not normal organs can be reservoirs for extremely potent cytotoxins. We used normal tissues of the central nervous system (CNS) and a number of peripheral human tissues using blots or electrophoretic gels with multiple samples of mRNA of variety of
(A) 140-kDa IL4R $\beta$-chain. (B) $45-\mathrm{kDa}$ IL13R $\alpha^{\prime}$ chain. (A) and (B) constitute the elements of the heterodimeric high affinity IL13/4R. (C) A 42-kDa monomer of IL13R $\alpha$.

organs. Our study offers a unique insight into the presence and biological distribution of the IL13 receptors. Unexpectedly, we identified IL13R $\alpha$ as a protein that belongs to a family of tumor antigens evoking usually prominent immune responses in cancer patients. Consequently, the HGG-associated IL13R $\alpha$ appears to represent a target for a variety of immunotherapeutic approaches, other than cytotoxins, for the treatment of human malignant gliomas.

\section{Materials and Methods}

Sources of RNA

High-grade glioma cell lines A-172 MG, U-373 MG, U-251 MG and human glioblastoma multiforme explant cells isolated in our laboratory, G-48, were grown in culture-appropriate media. Total RNA was extracted from the cells using the acid-guanidium isothiocyanate-phenolchloroform method (15). Poly(A)+ RNA was further isolated using the Mini-oligo(dT) Cellulose Spin Column Kit ( 5 prime $\rightarrow 3$ prime Inc., Boulder, CO). $2 \mu \mathrm{g}$ of Poly (A) + RNA was electrophoresed on a $1 \%$ agarose formaldehyde gel, transferred to $0.45 \mu \mathrm{m}$ magna charge nylon (MSI, Westborough, MA) and UV-crosslinked (Stratagene, La Jolla, CA). RNA-blotted membranes were also purchased from Clontech 
(Palo Alto, CA). Two Multiple Tissue Expression (MTE ${ }^{\mathrm{TM}}$ ) Blots (cat \# 7770-1 and \# 7775-1; Clontech) were analyzed to determine the tissue distribution of the IL13 binding proteins. Two sets of Human Brain Multiple Tissue Northern (MTNTM) Blots (cat \# 7755-1 and \# 7769-1; Clontech) were assayed to confirm the true presence of the transcripts. In addition, two Human Tissue Northern (MTNTM) Blots (cat \# 7759-1 and \# 7760-1; Clontech) were analyzed to verify the tissue distribution of the hIL13R $\alpha$ transcript.

\section{cDNA Probes}

cDNA probes were generated either by polymerase chain reaction (PCR; IL13R $\alpha$ and IL13R $\left.\alpha^{\prime}\right)$ or by restriction digest $\left(\mathrm{IL}_{4} \mathrm{R}_{\beta}=\mathrm{p} 140\right)$. cDNA containing human IL13R $\alpha$ was kindly provided by Dr. Pascual Ferrara of Sanofi Recherche, France, and the cDNA containing human IL13R $\alpha^{\prime}$ was a generous gift of Dr. Douglas J. Hilton of The Walter and Eliza Hall Institute of Medical Research, Australia, which also contained 93 bases of murine IL3. Plasmid pHuIL4R/ID was used to obtain a fragment of $\mathrm{IL}_{4} \mathbf{R}_{\beta}$ by the restriction digest. The fragments were electrophoresed on a $1 \%$ agarose gel, excised from the gel and purified using QIAquick Gel Extraction Kit (Qiagen Inc., Valencia, CA). Actin cDNA was purchased from Clontech Labs. The primers for human IL13R $\alpha$ were as follows:

forward 5'AAGATTTGGAAGCTTATGGCTTTCGTTTGC-3' reverse $5^{\prime}$ -

TCCCTCGAAGCTTCATGTATCACAGAAAAA-3'

The primers for human IL13R $\alpha^{\prime}$ were as follows:

forward 5'ATTATTAAGCTTATGGAGTGGCCGGCG-3' reverse $5^{\prime}$ -

TAACCGGAAGCTTCACTGAGAGGCTTT-3'

\section{Northern Blot Analysis}

Membranes were prehybridized overnight at $42^{\circ} \mathrm{C}$ in solution consisting of $50 \%$ formamide, $5 \mathrm{X}$ (SSC), $50 \mathrm{mM}$ sodium phosphate, $5 \mathrm{X}$ Denhardts, $50 \mu \mathrm{g} / \mathrm{ml}$ sheared salmon sperm DNA, and $1 \%$ (SDS). Membranes were subsequently hybridized overnight at $42^{\circ} \mathrm{C}$ in the same solu- tion with the addition of full-length cDNA probes labeled by random priming (Life Technologies, Rockville, MD) with ${ }^{32} \mathrm{P}$-dCTP using $1-2 \times 10^{6} \mathrm{cpm} / \mathrm{ml}$. Following hybridization, the membranes were washed with $2 \mathrm{X}$ SSC $/ 0.2 \%$ SDS at $42^{\circ} \mathrm{C}$ for $20 \mathrm{~min}$, followed by two washes with IX SSC/0.1\% SDS at $42^{\circ} \mathrm{C}$ for $20 \mathrm{~min}$ each. The membranes were exposed to autoradioraphic film X-OMAT AR (Eastman Kodak Co., Rochester, NY) and placed at $-80^{\circ} \mathrm{C}$ for 1,3 and 14 days. The membranes were subsequently stripped and reprobed up to three more times. The membranes were probed first with IL13R $\alpha$, followed by IL13R $\alpha^{\prime}$, $\mathrm{IL}_{4} \mathrm{R}_{\beta}=\mathrm{pl} 140$, and actin.

Films were scanned on a transparency scanner at a pixel size of $88 \times 88$ micron (Molecular Dynamics, Sunnyvale, CA). The images were compiled in Paint Shop Pro V 5.0 (Jasc software Inc., Eden Prairie, MN).

\section{Results \\ Northern Blot Analysis of Transcripts for IL13R $\alpha$ In Normal Organs}

IL13R $\alpha$ is a monomeric form of an IL4independent, IL13 receptor (Fig. 1C, and Fig. 2, closed drawing) that is associated with selected malignancies, including human malignant gliomas. It would, thus, be desirable for the purpose of exploiting this receptor for diagnosis, imaging and treatment of HGG, that IL13R $\alpha$ was expressed primarily in transformed tissues and not in healthy, unchanged organs. To first explore the expression of IL13R $\alpha$ in physiology, we performed extensive examination of the presence of transcripts for this protein among multiple normal tissues, including 20 discrete regions of the CNS and a variety of normal peripheral organs. All Northern blots using the same membranes were performed with respective labeled cDNAs in the following order: IL13R $\alpha$, IL13R $\alpha^{\prime}, \operatorname{IL}_{4} \mathbf{R}_{\beta}$ and $\beta$-actin. This assured us of not underestimating the levels of transcripts for IL13R $\alpha$ due to the usage of membranes with mRNA. Both the dot-blot analyses (not shown Clontech cat \# 7770-1 and 7775-1), and the electrophoretically separated transcripts for IL13R $\alpha$ (Fig. 2, panels I-IV) demonstrated mostly undetectable, or very weak signals in few cases, of IL13R $\alpha$ transcripts in the organs studied, even after 2-weeks of film exposure. However, with the first dot-blot developed in our laboratory (Clontech cat \# 7770-1; not 

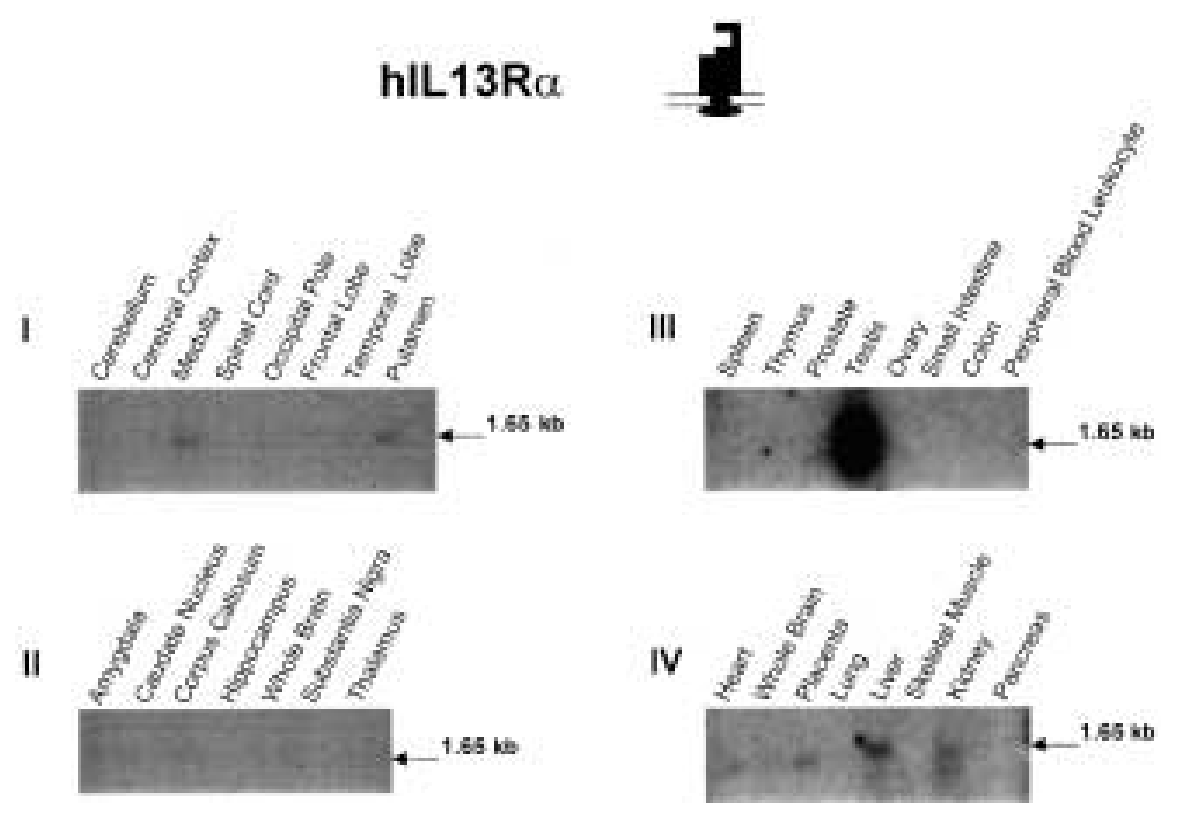

Fig. 2. Northern blot analysis of human (IL13R $\alpha$ ) transcripts (closed figure) in series of CNS (panels I and II) and peripheral tissues (panels III and IV). The migration position of mRNA is shown in kilobases. Films were exposed for 2 weeks.

shown) we noticed an unusually high density labeling with the IL13R $\alpha$ cDNA probe to transcripts derived from testis (dot l-D). This was also found using another Northern blot membrane (not shown; Clontech cat \# 7775-1; dot 8-F). Other organs, which transcripts hybridized to the IL13R $\alpha$ cDNA (aorta, liver, and pituitary gland), had the density of labeling much lower in dot-blots (2-C, 2-E, 4-D, and 4-B, 9-A, 3-D, respectively). Of importance, there was no evidence for the presence of significant IL13R $\alpha$ expression in the CNS (blot \# 7775-1, columns 13; and blot \# 7770-1, rows A and B).

To confirm the true positivity of our findings made using dot-blot analysis, both qualitatively and size-wise, we used membranes with electrophoretically separated mRNAs. Again, the discrete regions of normal human brain did not produce clear-cut hybridization signals (Fig. 2, panels I and II). On the other hand, the only organ with the prominent hybridization band corresponding to the mRNA of $1.5 \mathrm{~kb}$ was seen in testis (Fig. 2, panel III). Others also found this size of the transcript for IL13R $\alpha$ (16). Poorly detectable signals were seen in placenta, liver, and kidney (Fig. 2, panel IV). Thus, only the testes can be considered as the prominent expressors of IL13R $\alpha$ among normal tissues. No transcripts for IL13R $\alpha$ were readily detected in the CNS.
Northern Blot Analysis of Transcripts for IL13R $\alpha^{\prime}$ In Normal Tissues

IL13R $\alpha^{\prime}$ is a component of a heterodimeric form of IL13 receptor that is shared with IL4, IL13/4 receptor (Fig. 1B and Fig. 3, closed drawing). This shared IL13/4 receptor is the only physiological signaling IL13 receptor (5), although we find it at modest levels in several adenocarcinomas as well (4). However, we consider IL13R $\alpha$ a much better molecular target when compared with the IL13/4 for improving the management of diseases, such as HGG. This is primarily because IL-4 independent IL13 binding densities are much higher in malignancy, compared with IL13/4 sites $(4,9)$. We also produced evidence suggesting a feasibility to redirect IL13 from IL13/4 receptor sites towards IL4-independent sites, such as IL13R $\alpha(12,13)$. We, thus, explored in detail the expression of IL13R $\alpha^{\prime}$ in normal human tissues (Fig. 3). Either dot-blot analyses (not shown) or electrophoretically separated transcripts (Fig. 3, panels I-IV) unequivocally demonstrated IL13R $\alpha^{\prime}$ hybridization in a variety of the organs studied. The most enriched in the IL13R $\alpha^{\prime}$ transcripts were medulla, spinal cord, substantia nigra, thalamus, and in corpus callosum, within the CNS (blot \# 7770-1, dots 2-C, 2-G, 3-A, 3-C, 3-E; respectively). Size fractionated mRNAs confirmed that there were 


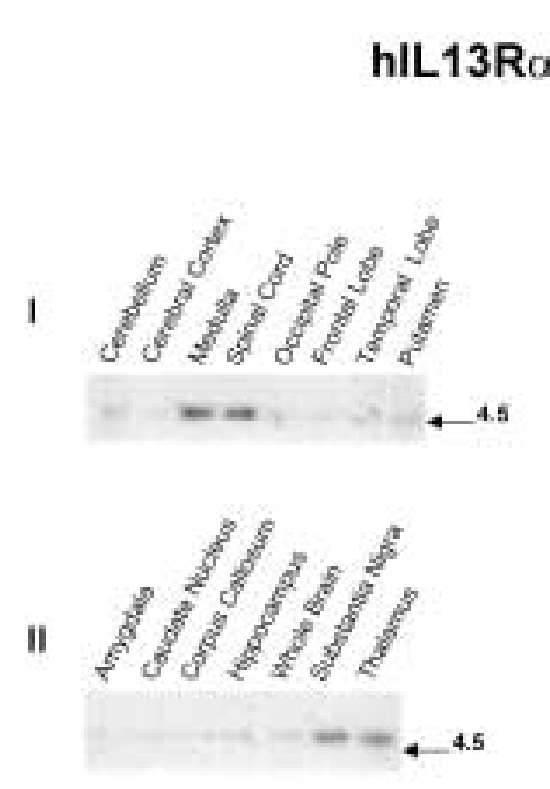

Fig. 3. Northern blot analysis of human interleukin 13 receptor $\alpha$ (IL13R $\left.\alpha^{\prime}\right)$ transcripts (closed figure) in series of CNS (panels I and II) and peripheral tissues (panels III and IV). The

many positive signals seen in dot-blots, with the strongest signals observed in ovary, heart, liver and kidney (Fig. 3, panels III and IV). Of interest, liver showed two hybridized species of mRNA (one of $4.5 \mathrm{~kb}$ and the other of 2.0 $\mathrm{kb}$ ) as an example of a normal organ with a doublet of positive signals of different sizes. In summary, discrete regions of normal human brain did produce clear-cut positive hybridization signals for IL13R $\alpha^{\prime}$ (Fig. 3, panels I and II). In addition, many vital peripheral organs exhibited hybridization bands corresponding to the mRNA of $4.5-4.65 \mathrm{~kb}$ (Fig. 3, panels III and IV).

Gene Expression Analysis of $I L 4 R_{\beta}$ In Normal Tissues

$\mathrm{IL}_{4} \mathbf{R}_{\beta}$ is another component of a heterodimeric form of IL13 receptor, besides IL13R $\alpha^{\prime}$, that is shared with IL4, IL13/4 receptor (Fig. 1A and Fig. 4, closed drawing). Thus, we analyzed whether the distribution of IL $_{4} \mathbf{R}_{\beta}$ gene expression pairs the expression of that for IL13R $\alpha^{\prime}$, its partner in an IL13/4 signaling receptor (Fig. 1). All Northern blot analysis membranes used in this study demonstrated enriched content of the $\operatorname{IL}_{4} \mathrm{R}_{\beta}$ transcripts in a variety of tissues

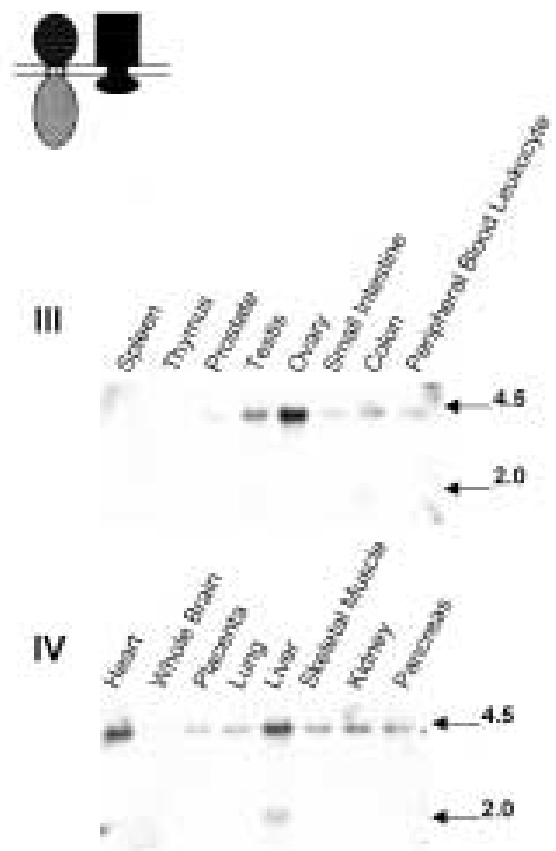

migration position of mRNA is shown in kilobases. Films were exposed for 2 weeks, except for membranes shown in panels III and IV, which were exposed for 3 days.

(Fig. 4, panels I, II, and IV). The presence of the transcripts within the CNS was most evident, as was the case for IL13R $\alpha^{\prime}$, in medulla, spinal cord, substantia nigra and thalamus (blot \# 7775-1, dots 2-G, 3-E, 3-A, 3-C and blot \# 7770-1, dots 8-A, 7-B, 3-B, 5-B, not shown) (Fig. 4, panels I and II). Among normal peripheral organs, liver, lung, kidney, intestinal tract, spleen, stomach, and testis demonstrated gene expression of IL4R $\mathbf{R}_{\beta}$, which was generally similar to that seen with IL13R $\alpha^{\prime}$ (not shown). Thus, discrete regions of normal human brain contain transcripts for both IL13R $\alpha^{\prime}$ and IL4R $R_{\beta}$, a complete heterodimer of the shared IL13/4 receptor. Furthermore, several vital peripheral organs contained the two subunits of the IL13/4 receptor, including heart, liver, lung and intestinal tract.

\section{Control Hybridization of $\beta$-actin}

All membranes used for Northern blot analysis of IL13 receptors transcripts were also hybridized with a cDNA probe for a housekeeping gene, $\beta$-actin (Fig. 5 ; dot blots and panel III not shown). The intensity of the signals for $\beta$-actin usually was in accordance to the amount of mRNA present on the membranes, as estimated by the manufacturer. 


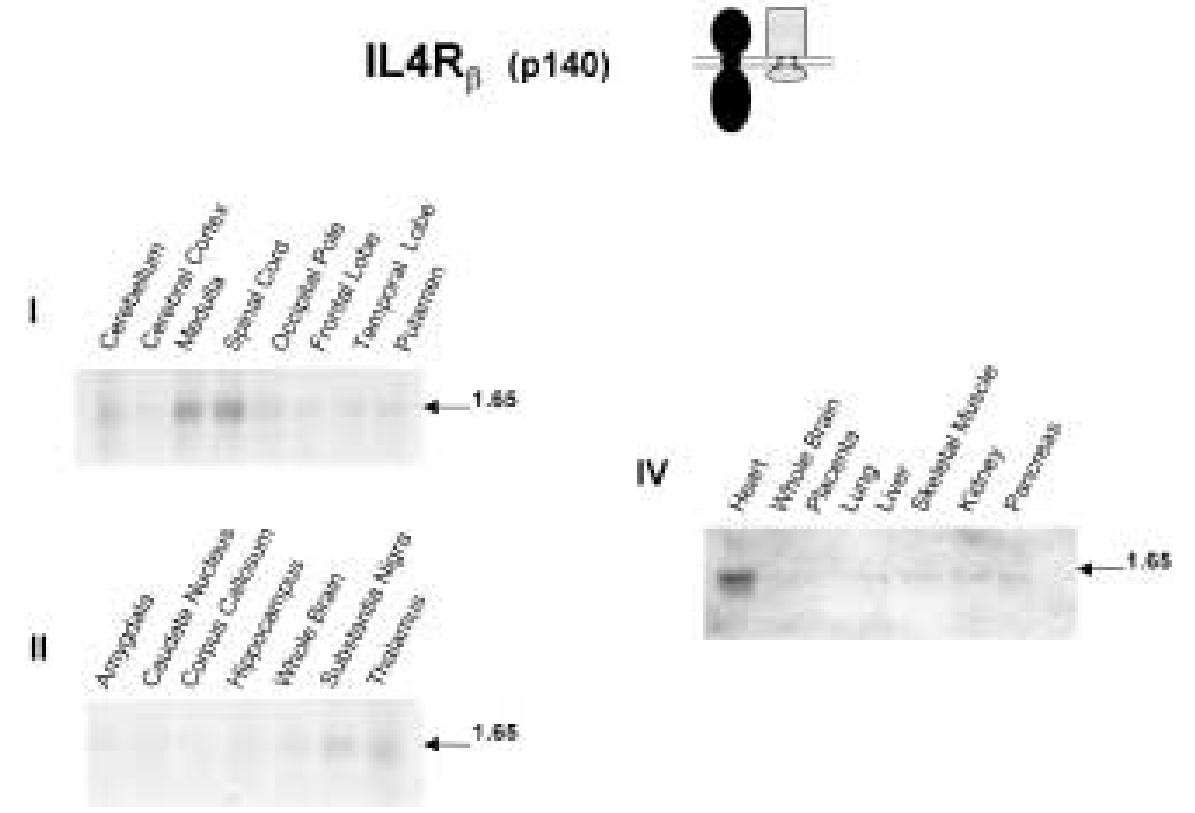

Fig. 4. Northern blot analysis of human 140-kDa interleukin 4 receptor (IL4R) $\beta$-chain transcripts (closed figure) in series of CNS (panels I and II) and peripheral tissues (panel IV). The migration position of mRNA is shown in kilobases. Films were exposed for 2 weeks.

Gene Expression of IL13 Receptors In Cells

We next analyzed gene expression of the two IL13 receptors in malignant and normal cells (Fig. 6). Transcripts for IL13R $\alpha, \operatorname{IL} 13 R \alpha^{\prime}, \operatorname{IL}_{4} \mathrm{R}_{\beta}$ and $\beta$-actin were examined in serial hybridization assays. Our laboratory's isolated explant cells of HGG (G-48), as well as human malignant glioma established cells (A-172 MG, U-373 MG, and U-25 I MG), demonstrated intense signals for IL13R $\alpha$ (Fig. 6). On the other hand, the transcripts for the elements of the shared IL13/4 receptor, IL13R $\alpha^{\prime}$ and IL4R $_{\beta}$,

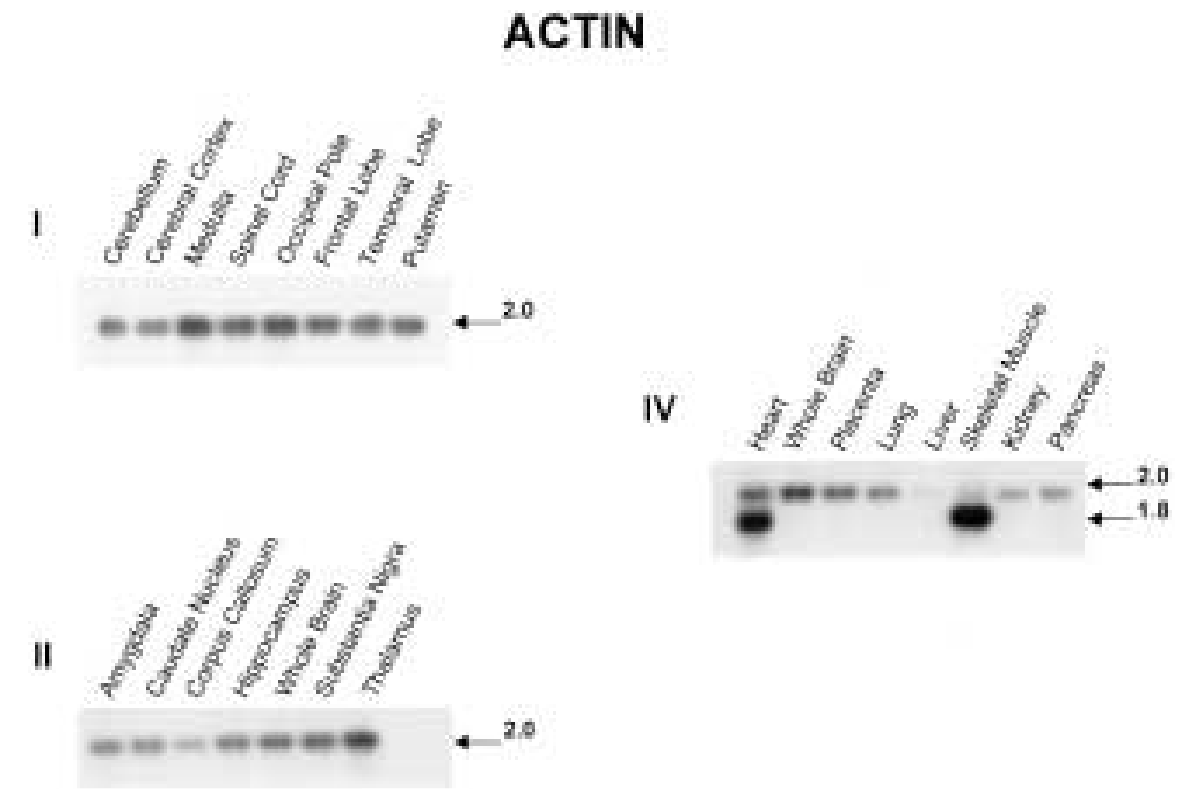

Fig. 5. Northern blot analysis of human $\beta$-actin transcripts in central nervous system (CNS) (panels I and II) and peripheral tissues (panel IV). The migration position of mRNA is shown in kilobases. Films were exposed for $1-3 \mathrm{hr}$. 


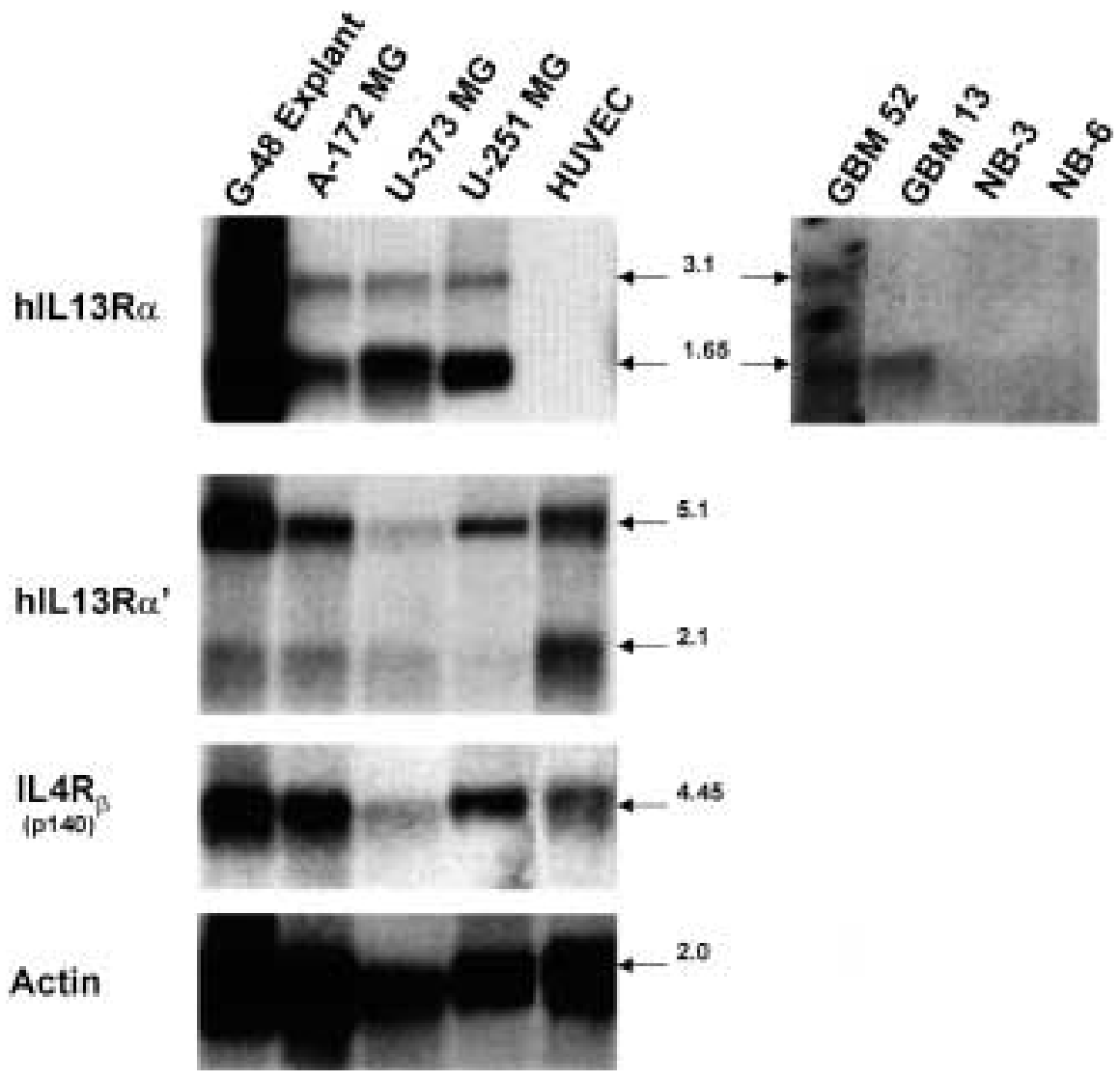

Fig. 6. Northern blot analysis of interleukin 13 (IL13) receptors transcripts in malignant glioma cells (G-48, A-172 MG, U-373 MG, and U-25 1 MG), normal human umbilical vein endothelial cells (HUVEC) and in surgical specimens of high-grade glioma (HGG) and normal

were found at lower levels, compared with that for IL13R $\alpha$ (Fig. 6). A-172 MG cells appeared to be the most enriched in the components of the IL13/4 receptor heterodimer. This result is a molecular blueprint for the observed phenomenon of a higher density of IL4-independent IL13 binding sites vs. the sites shared with IL4 found in HGG (9). Of interest, two species of different sizes of the transcripts for both ILI3R $\alpha$ and IL13R $\alpha^{\prime}$ were seen in cells (Fig. 6). In a control assay, human umbilical vein endothelial cells (HUVEC) showed the presence of transcripts for IL13R $\alpha^{\prime}$ and IL4R $R_{\beta}$, but not for IL13R $\alpha$ (Fig. 6). The elements of the shared IL13/4 receptor were demonstrated in HUVEC previously (17). This is in agreement with our data indicating that A-172 MG cells are responsive to the IL4-based cytotoxin in a manner similar to that seen in HUVEC (9; and Debinski, unpublished). human brain. The analysis was conducted using membranes blots developed in house, as described in Methods section. The migration position of mRNA is shown in kilobases. Films were exposed for 2 weeks, except for actin (1 hr).

In our preliminary evaluation, we detected gene expression of IL13R $\alpha$ in two specimens of HGG (Fig. 6, GBM 13 and GBM 52), but we could not demonstrate it in two normal brain specimens (Fig. 6, NB 3 and NB 6). However, the transcripts for IL13R $\alpha^{\prime}$ were found in all of these specimens (not shown).

\section{Discussion}

The presence of the form of IL13 receptor, IL13R $\alpha$, that is IL4-independent in series of discrete regions of the CNS and in many normal organs was found to be negligible. Only the testes showed an intense signal for the IL13R $\alpha$ message, using Northern blots analysis. On the other hand, the mRNA for IL13R $\alpha$ was readily detected in human glioma cells. Unlike IL13R $\alpha$, the message for the two components of the shared IL13/4R, IL13R $\alpha^{\prime}$ and 
IL4 $R_{\beta}$, was readily found in several regions of the CNS, such as medulla, spinal cord, substantia nigra and thalamus, and in vital peripheral organs, such as lung, liver, kidney, heart, and gastrointestinal tract. The intensity and the localization of the signal for IL13R $\alpha^{\prime}$ paired in principal with that for IL $4 R_{\beta}$, which indicates the presence of a functional signaling heterodimer of the IL13/4 receptor in several regions of the normal human brain. These results support our attempts at redirecting potent antiglioma diagnostics/therapeutics away from the physiological IL13/4R and more towards the restrictive, IL4-independent, receptor for IL13 $(12,13,18)$. This molecular maneuver provides a significant increase in the specificity of molecular targeting of human malignant gliomas. Even for a local intracranial interstitial delivery of IL13-based therapeutics (14), it would be preferable to employ reagents with altered reactivity towards IL13/4R, since normal human brain evidently possesses this receptor (Figs. 3 and 4).

Our current study also revealed, unexpectedly, another novel and intriguing aspect of the IL13 system's pathobiology. We documented previously that the binding sites for IL13 that are IL4-independent are high-grade, gliomaassociated receptor sites. In addition, the gene for IL13R $\alpha$, an IL4-independent IL13-binding protein, is localized to chromosome $X$ (16). Furthermore, our present study demonstrates that the IL13R $\alpha$ expression among normal tissues is prominent only in the testes. This triumvirate of factors mimics the three main biologic characteristics of a growing family of serological identification of antigen by recombinant expression cloning (SEREX) tumor antigens $(19,20)$. Such antigens have been uncovered by the procedure in which sera of cancer patients serve as a source of autologous anti-tumor antibodies and phage libraries of patients' tumors serve as a source of tumor antigens $(19,21)$. Since almost all of the SEREX antigens showed a prominent expression in testes, with their very limited presence in few other normal tissues, a new term of testis/cancer antigens was proposed. The methodology was simplified from using libraries based on patients' tumors to solely testes-derived libraries, with subsequent successful identification of new tumor antigens (22). Of interest, the testis/cancer tumor antigens are representative of the group of antigens which were originally $\mathrm{T}$ cell-defined antigens, such as MAGE,
GAGE, BAGE, etc., and which were most successfully employed in anti-cancer vaccines in the clinic thus far (23).

The search of the SEREX database (www.licr.org, University of Saarland, Homburg, Germany) failed to match IL13R $\alpha$ with any known SEREX or testis/cancer antigen. This may be due to several reasons. First, the patients with HGG are considered to have a depressed immune system; therefore, it may be difficult to isolate the existent autologous anti-glioma antibodies due to their possible low titers (24). However, the evidence for testis/cancer antigens being expressed in a subfraction of brain tumors has been provided recently (22). In addition, MAGE and GAGE tumor antigens have been shown to be present in HGG to some extent by their transcript amplification (25). Second, the recombinant expression cloning involves expression of human proteins in a prokaryotic host using a phage system $(19,22)$. Our own experience with the IL13R $\alpha$ expression in bacteria has been, thus far, negative (unpublished data). It is plausible that the original SEREX detection system fails to recognize some of the existing tumor antigens on the basis of their inability to refold properly in a phage presentation system. This scenario could be especially true for transmembrane proteins. Third, investigations exploring SEREX have not come up naturally with such an antigen yet. Independent of all of these three possibilities, it is becoming imperative to screen HGG patients for the presence of autologous anti-IL13R $\alpha$ antibodies and to examine $\mathrm{T}$-cell responses by IL13R $\alpha$. If found, then it would be of interest to correlate the titer of autologous anti-IL13R $\alpha$ antibodies with the stage and the course of highgrade gliomas. We have commenced studies to address those new and important issues.

Cytotoxins based on IL13 mutants are extremely potent anti-HGG compounds in vitro and in vivo $(1,10)$. We provided evidence for a ubiquitous over-expression of an IL13R that is IL4-independent in human HGG specimens (1). Our concomitant analysis of gene expression of the two IL13 receptor types underlines the need to avoid the targeting of the shared IL13/4R in experimental diagnostic/ therapeutic approaches that are related to HGG. In addition, the presented pathological and biological distribution of the IL13 receptors enabled us to identify the more restrictive HGG-associated receptor for IL13 as a previously unknown testis/cancer antigen, the first 
of such prevalence among brain tumors. This opens an attractive possibility of IL13R $\alpha$ also becoming a rational target for treatments, such as protein/peptide-based vaccines for the treatment of malignant gliomas (23). We envision that a combination of direct cytotoxic therapy and active immunotherapy of human HGG would be a desirable molecular therapeutic approach for this invariably incurable disease (26). Cancer/testis antigens have been suggested to play a major role in new immunotherapies of cancer (27). Moreover, such therapeutic opportunities could be available for patients with other cancers or diseases, such as the ones over-expressing IL $13 R \alpha$, since normal tissue representation of this receptor is negligible. We recently underlined a possible significance of the presence of testis/cancer antigens in brain tumors (28).

\section{Acknowledgments}

This work was supported by the Surgery Feasibility Grant and NIH grants R01 CA74145.

\section{References}

1. Debinski W, Gibo D, Hulet SW, Connor JR, Gillespie GY. (1999) Receptor for interleukin 13 is a marker and therapeutic target for human high-grade gliomas. Clin. Cancer Res. 5: 985-990.

2. McKenzie ANJ, Culpepper JR, De Waal Malefyt $\mathrm{R}$, et al. (1993) Interleukin 13, a T-cell-derived cytokine that regulates human monocyte and B-cell function. Proc. Natl. Acad. Sci. 90: 37353739.

3. Minty A, Chalon P, Derocq JM, et al. (1993) Interleukin-13 is a new human lymphokine regulating inflammatory and immune responses. $\mathrm{Na}$ ture 36: 248-251.

4. Debinski W, Obiri NI, Pastan I, Puri RK. (1995) A novel chimeric protein composed of interleukin 13 and Pseudomonas exotoxin is highly cytotoxic to human carcinoma cells expressing receptors for interleukin 13 and interleukin 4. J. Biol. Chem. 270: 16775-16780.

5. Obiri NI, Leland P, Murata T, Debinski W, Puri RK. (1997) The IL-13 receptor structure differs on various cell types and may share more than one component with IL-4 receptor. J. Immunol. 158: $756-764$.

6. Hilton DJ, Zhang J-G, Metcalf D, Akexander WS, Nicola NA, Wilson TA. (1996) Cloning and characterization of a binding subunit of the interleukin 13 receptor that is also a component of the interleukin 4 receptor. Proc. Natl. Acad. Sci. U.S.A. 93: 497-501.
7. Idzerda RL, March CJ, Mosley B, et al. (1990) Human interleukin-4 receptor confers biological responsiveness and defines a novel receptor superfamily. J. Exp. Med. 171: 861-873.

8. Debinski W, Obiri NI, Powers SK, Pastan I, Puri RK. (1995) Human glioma cells over-express receptor for interleukin 13 and are extremely sensitive to a novel chimeric protein composed of interleukin 13 and Pseudomonas exotoxin. Clin. Cancer Res. 1: 1253-1258.

9. Debinski W, Miner R, Leland P, Obiri NI, Puri RK. (1996) Receptor for IL 13 does not interact with IL4 but receptor for IL4 interacts with IL13 on human glioma cells. J. Biol. Chem. 271: 22428-22433.

10. Debinski W. (1998) An immune regulatory cytokine and high-grade gliomas: An unexpected link. Critic. Rev. Oncogen. 9: 256-268.

11. Caput D, Laurent P, Kaghad M, et al. (1996) Cloning and characterization of a specific interleukin (IL)-13 binding protein structurally related to the IL-5 receptor $\alpha$ chain. J. Biol. Chem. 271: 16921-16926.

12. Debinski W, Gibo DM, Obiri NI, Kealiher A, Puri RK. (1998) Novel anti-brain tumor cytotoxins specific for cancer cells. Nature Biotech. 16: 449-453.

13. Thompson J, Debinski W. (1999) Mutants of interleukin 13 with altered reactivity toward IL13 receptors. J. Biol. Chem. 274: 29944-29950.

14. Laske DW, Youle RJ, Oldfield EH. (1997) Tumor regression with regional distribution of the targeted toxin TF-CRM107 in patients with malignant brain tumors. Nature Med. 3: 13621368.

15. Chomczynski P, Sacchi N. (1987) Single-step method of RNA isolation by acid guanidinium thiocyanate-phenol-chloroform extraction. Anal. Biochem. 162: 156-159.

16. Guo J, Apiou F, Mellerin M-P, Lebeau B, Jacques Y, Minvielle S. (1997) Chromosome mapping and expression of the human interleukin-13 receptor. Genomics 42: 141-145.

17. Schnyder B, Lugli S, Feng N, et al. (1996) Interleukin-4 (IL-4) and IL-13 bind to a shared heterodimeric complex on endothelial cells mediating vascular cell adhesion molecule- 1 induction in the absence of the common $\gamma$ chain. Blood 87: 4286-4295.

18. Debinski W, Thompson J. (1999) Retargeting interleukin 13 for radioimmunodetection and redioimmunotherapy of human high-grade gliomas. Clin. Cancer Res. 5: 3143s-3147s.

19. Sahin U, Tureci O, Schmitt H, et al. (1995) Human tumors elicit multiple specific immune response in autologous host. Proc. Natl. Acad. Sci. 92: 11810-11813.

20. Tureci O, Sahin U, Zwick C, Koslowski M, Seitz G, Pfreundschuh M. (1998) Identification of a meiosis-specific protein as new member of 
the cancer/testis antigen superfamily. Proc. Natl. Acad. Sci. 95: 5211-5216.

21. Scanlan MJ, Gordan J, Williamson B, et al. (1999) Antigens recognized by autologous antibody in patients with renal-cell carcinoma. Int. $J$. Cancer 83: 456-464.

22. Tureci O, Sahin U, Zwick C, Neumann F, Pfreundschuh M. (1999) Exploitation of the antibody repertoire of cancer patients for the identification of human tumor antigens. Hybridoma 18: 23-28.

23. Rosenberg SA, Yang JC, Schwartzentruber DJ, et al. (1998) Immunologic and therapeutic evaluation of a synthetic peptide vaccine for the treatment of patients with metastatic melanoma. Nature Med. 4: 321-327.

24. Mahaley MS, Brooks WH, Roszman TL, Bigner DD, Duka L, Richardson S. (1977) Immunobiology of primary intracranial tumors. J. Neurosurg. 46: 467-476.
25. Scarcella DL, Chow CW, Gonzales MF, Economou C, Brasseur F, Ashley DM. (1999) Expression of MAGE and GAGE in high-grade brain tumors: A potential target for specific immunotherapy and diagnostic markers. Clin. Cancer Res. 5: 335-341.

26. Davis FG, Freels S, Grutsch J, Barlas S, Brem S. (1998) Survival rates in patients with primary malignant brain tumors stratified by patient age and tumor histological type: an analysis based on Surveillance, Epidemiology, and End Results (SEER) data, 1973-1991. J. Neurosurgery 88: 1-10.

27. Rosenberg SA. (1999) A new era for cancer immunotherapy based on the genes that encode cancer antigens. Immunity 10: 281-287.

28. Mintz A, Debinski W. (2000) Cancer genetics/epigenetics and the $\mathrm{X}$ chromosome: Possible new links for malignant glioma pathogenesis and immune-based therapies. Crit. Rev. Oncogen 11: 77-95. 